\title{
Germanica
}

\section{Robert Neumanns Hochstaplernovelle - Versuch über eine gescheiterte Karriere}

La «Nouvelle de l'importeur» de Robert Neuman. Essai sur l'échec d'une carrière

\section{Alfred Strasser}

\section{OpenEdition}

\section{Journals}

Édition électronique

URL : http://journals.openedition.org/germanica/1787

DOI : 10.4000/germanica. 1787

ISSN : 2107-0784

\section{Éditeur}

Université de Lille

\section{Édition imprimée}

Date de publication : 31 décembre 2004

Pagination : 81-89

ISBN : 9782913857148

ISSN : 0984-2632

Référence électronique

Alfred Strasser, "Robert Neumanns Hochstaplernovelle - Versuch über eine gescheiterte Karriere »,

Germanica [Online], 35 | 2004, Online erschienen am: 05 Oktober 2012, abgerufen am 06 Oktober 2020. URL : http://journals.openedition.org/germanica/1787 ; DOI : https://doi.org/10.4000/germanica.1787

Ce document a été généré automatiquement le 6 octobre 2020.

(c) Tous droits réservés 


\section{Robert Neumanns Hochstaplernovelle - Versuch über eine gescheiterte Karriere}

La «Nouvelle de l'importeur» de Robert Neuman. Essai sur l'échec d'une carrière

Alfred Strasser

1 Als der Münchener Kurt Desch Verlag im Jahre 1961 Robert Neumanns neuen Roman Olympia ${ }^{1}$ als « Ergänzung und heitere Fortsetzung 》 von Thomas Manns Bekenntnisse des Hochstaplers Felix Krull ankündigte, reagierte Thomas Manns Tochter Erika sofort sehr heftig auf diese Verlagswerbung. Sie bezichtigte Neumann nicht nur Plagiator ihres Vaters zu sein, sondern erwirkte außerdem per Gerichtsbeschluß, daß der Verlag diese Reklame sofort zurückziehen mußte ${ }^{2}$. Die Sache war damit aber noch nicht bereinigt : Nach der Auslieferung des Thomas Mann gewidmeten Romans bemühte Erika Mann auch noch den S. Fischer-Verlag, gerichtlich gegen die Verwendung von Thomas Manns Figur vorzugehen, denn Neumanns Praxis sei ein «Einbruch in die Persönlichkeitsrechte einer Mann'schen Romanfigur $»^{3}$, so Erika Mann. In einer nicht besonders sensibel geführten außergerichtlichen Polemik zwischen Erika Mann und Robert Neumann forderte diese dann Neumann auf, entweder den Roman umzuschreiben oder ihn ganz zurückzuziehen. Die Auseinandersetzung endete schließlich mit einem weiteren Gerichtsbeschluß, in dem Neumann dazu verurteilt wurde, 19 Zeilen seines Romans abzuändern.

2 Robert Neumanns Verteidiger wiesen unter anderem Erika Manns Plagiatsvorwürfe mit dem Argument zurück, daß ursprünglich Thomas Mann für seinen Roman Bekenntnisse des Hochstaplers Felix Krull Motive von Neumann übernommen habe, denn Neumann hätte schon 1930 mit der Hochstaplernovelle einen Text vorgelegt, in dem die Figur des Hochstaplers thematisiert ist.

3 Dem ist entgegenzuhalten, daß jedoch Thomas Mann bereits 1911 an seinem Roman arbeitete und im selben Jahr ein erster Teil, Das 25. Jahr, in einem Almanach des FischerVerlages erschien. Dem folgten 1922 (Buch der Kindheit) ${ }^{4}$ und 1937 zwei weitere Teile. 
Thomas Mann hatte also diesen Stoff schon bearbeitet, als Neumann gerade vierzehn Jahre alt war.

4 Robert Neumann versuchte schließlich den Streit beizulegen, indem er im Nachwort des Sammelbandes Karrieren erklärte, daß ein Schriftsteller wie Thomas «Mann die Inspiration durch [ihn] noch weniger nötig [hat] als [er] die durch Mann $»^{5}$, doch eine Figur wie die des Hochstaplers bringe es naturgemäß mit sich, daß in dem jeweiligen Text zweier Autoren über ein solches Thema notgedrungen identische oder ähnliche Motive vorkämen.

Zwei Jahre nach dieser Erklärung revanchiert sich Robert Neumann auf seine Art für die erlittene Niederlage : Er veröffentlichte 1968 in seinem Buch Vielleicht das Heitere. Tagebuch aus einem anderen Jahr den Ausschnitt eines Briefs von Thomas Mann an Hanns Johst, den Erika Mann in der von ihr besorgten dreibändigen Auswahl der Briefe ihres Vaters ${ }^{6}$ nicht aufgenommen hatte. In diesem Brief vom 16. 9. 1920, den man, nach Neumann, kennen müsse, wenn man zu Thomas Mann ja sagen wolle, bedankte sich dieser bei Johst für eine Büchersendung. Darin heißt es unter anderem :

Ich liebe Sie sehr, Herr Hanns Johst, und freue mich Ihres Daseins. Sie stellen Jugend dar, Kühnheit, Radikalismus, stärkste Gegenwart - ohne irgendetwas mit jener gallojüdischeninternationalistischen ,Geistigkeit' zu schaffen zu haben, von der das deutsche Geistesleben sich eine Weile tyrannisieren lassen zu müssen glaubte...

6 Nun war Hanns Johst nicht irgendein Schriftsteller : Er begann als expressionistischer Dramatiker, machte aber um 1920 eine Wendung zum deutschnationalen Lager durch und wurde zu einem der fanatischsten nationalsozialistischen deutschen Autoren. Als Präsident der Reichsschrifttumskammer war er, der Freund Heinrich Himmlers, den er im « Kampfbund für deutsche Kultur» kennengelernt hatte, zwischen 1935 und 1945 für die Säuberung des deutschen Schrifttums von "artfremden» und «volksschädlichen» Schriftstellern verantwortlich. Einem mißliebigen Schriftstellerkollegen drohte er gewöhnlich damit, ihn « einmal 4 Wochen KZ genießen zu lassen $»^{8}$, wenn er nicht schnellstens eine parteikonforme Gesinnung einnähme.

7 Johsts Haltung Thomas Mann gegenüber hatte sich seit dem Briefwechsel von 1920 gründlich geändert : 1933 schickte der spätere Präsident der Reichsschrifttumskammer Thomas Mann keine Widmungsexemplare seiner Erstausgaben mehr, sondern er schlug im September seinem Freund Himmler vor, ihn inhaftieren zu lassen und so über ein Druckmittel gegen seinen Sohn Klaus zu verfügen. Dadurch könnte vielleicht Klaus Mann dazu gebracht werden, seine «antideutsche « Publikationstätigkeit ${ }^{9}$ aufzugeben ${ }^{10}$. Auch wenn Erika Mann vermeiden wollte, daß Thomas Manns antifranzösische und antisemitische Ressentiments nach dem Ersten Weltkrieg bekannt werden, ist es angesichts seiner Entwicklung in der NS-Zeit unverständlich, daß sie den Brief ihres Vaters an Hanns Johst verheimlichen wollte. Es ist aber auch nicht ganz von der Hand zu weisen, daß es sich bei der von Neumann vom Zaun gebrochenen Polemik um diesen Brief um einen Racheakt für die erwähnten gerichtlichen Schritte handeln könnte.

Tatsächlich geht Robert Neumanns Hochstaplernovelle auf ein Projekt des Autors aus dem Jahre 1927 zurück. Nach dem Erscheinen seiner Parodiensammlung Mit fremden 
Federn, mit der ihm sein literarischer Durchbruch gelungen war, wollte er, um nicht definitiv als Autor eines bestimmten literarischen Genres abgestempelt zu werden, Texte in einer anderen Gattung publizieren. Neumann faßte deshalb den Plan, unter dem Titel «Karrieren » einen Zyklus von zehn Romanen und Novellen zu schreiben, von denen er aber nur sechs vollendete ${ }^{11}$. Aus der Perspektive eines Ich-Erzählers sollten dabei Figuren wie Hochstapler, Politiker, Klofrauen und andere "von innen her " beleuchtet werden, wobei der Autor die charakteristischen Merkmale der jeweiligen Person so sehr steigern wollte, daß sie "ins Absurde» kippen. Die Arbeitsmethode, " andere Personen zu impersonieren » Eine Persan auf allgemeine Charakterzüge $\mathrm{zu}$ reduzieren und einen allgemeingültigen Typus $\mathrm{zu}$ schaffen, sieht Neumann als eine schauspielerische, ja hochstaplerische Leistung des Schriftstellers (397), ganz im Sinne von Friedrich Nietzsche, der im Schauspieler den Prototyp jedes Künstlers sieht.

Der Schauspieler kann zuletzt auch beim tiefsten Schmerz nicht aufhören, an den Eindruck seiner Person und den gesamten szenischen Effekt zu denken [...] Der Heuchler, welcher immer ein und dieselbe Rolle spielt, hört zuletzt auf, Heuchler zu sein [...] Der Beruf fast jedes Menschen, sogar des Künstlers, beginnt mit Heuchelei, mit einem Nachmenschen von außen her, mit einem Kopieren des

Wirkungsvollen ${ }^{12}$.

Genau diese von Nietzsche in Menschliches, Allzumenschliches (1878/79) dargestellte Charakteristik trifft die wesentlichen Merkmale des Hochstaplers, der den «Schein zum Sein » macht. Da er seine Rolle unter allen Umständen immer weiter zu spielen hat, und er besonders auf Wirkung bedacht sein muß, damit er von den anderen Mitgliedern jener Gesellschaft, der er unbedingt angehören will, akzeptiert und beachtet wird, verinnerlicht er diese Rolle in dem Maße, daß sie ein Teil seiner Persönlichkeit wird. Auch Neumann als Autor schlüpft in eine Rolle, nämlich in die des Ich-Erzählers seiner Hauptfiguren im Karrieren-Zyklus. Diese seine Haltung hat der Autor aber auch auf die seiner Figuren übertragen, weswegen der Zyklus notwendigerweise mit einer Novelle über einen Hochstapler beginnt und dieser als einzige Figur noch ein zweites Mal in der Reihe thematisiert ist.

Der Aufbau der Novelle $^{13}$, eine Aneinanderreihung von drei verschieden langen, chronologisch unmittelbar aufeinander folgenden Episoden aus dem Leben des IchErzählers, die dieser linear erzählt, entspricht ganz der Intention des Autors zu « impersonieren ». Bereits im nur zwei Seiten langen ersten Teil werden die Licht und Schattenseiten des Hochstaplerdaseins deutlich, das der Ich-Erzähler distanziert als ein " ein wenig berauschendes, ein wenig lächerliches, flüchtig vergoldetes und zwiegesichtiges Abenteuer » (7) charakterisiert. Der Ich-Erzähler, als irgendein Baron X in einem Hotel « Des Anglais » in Juan les Pins abgestiegen, wird von seiner Partnerin Denise verlassen und kann deswegen seine Rolle als reicher Adeliger nicht mehr weiter spielen : Er muß der Hoteldirektion gegenüber einräumen, ohne Geldmittel zu sein. Als Bezahlung für seinen Hotelaufenthalt kann er nur die Garderobe seiner geflohenen Partnerin anbieten. Zwar gelingt es ihm, diese Situation ohne größere materielle Verluste zu bereinigen, aber es ist dennoch der schlimmste aller möglichen Zwischenfälle eingetreten : Er hat seine Rolle nicht mehr weiterspielen können, « die 
Sache war publik. [Er] konnte sich in dem Jahr zwischen Menton und St. Tropez nicht mehr blikken lassen. » (8)

Die Existenz des Ich-Erzählers spielt sich also ab zwischen dem Willen, als anerkanntes Mitglied in der höheren Gesellschaft zu glänzen und von dieser zu profitieren, und der unausgesprochenen Angst, als Hochstapler entdeckt zu werden. Der Zwischenfall an der Côte d'Azur veranlaßt ihn aber keineswegs, seinen Lebenswandel grundsätzlich in Frage zu stellen, sondern er zieht daraus nur die Lehre, mit Denise nie mehr zusammenzuarbeiten und bricht nach Venedig auf, nachdem ihm die Hoteldirektion aus Angst vor einem Skandal eine Zugfahrkarte dorthin finanziert hat. Wie stark sein Bewusstsein für die Zugehörigkeit zur höheren Gesellschaft ausgeprägt ist, zeigt seine Befindlichkeit während der Bahnreise, wo er in einem Abteil zweiter Klasse «mit Kammerdienern und Putzmacherinnen » (8) hat reisen müssen - eine für ihn absolut unerträgliche Situation.

In Venedig wiederholt sich schließlich der gleiche Zyklus : Der Erzähler steigt im ersten Hotel auf dem Lido als Mr. Davis ab, tritt hier aber nicht mit einer Begleiterin auf und wird durch eine Verwechselung für den abgereisten Lord Chesterton gehalten. Als solcher sucht er den Umgang mit den anderen Bewohnern des Hotels, wird schließlich in einen offensichtlich illustren kleinen Kreis sehr reicher Menschen aufgenommen und nutzt diese Stellung aus, um durch Wetten und Betrügereien zu Bargeld und Schmuck zu kommen. $\mathrm{Zu}$ guter Letzt wird er von einem der Hotelportiers, der ihn schon von früher her kennt, als Hochstapler entlarvt. Der Portier fordert den zwar lautstark protestierenden angeblichen Lord Chesterton auf, das Hotel schnellstens heimlich zu verlassen, auch diesmal wieder mit der Begründung, daß man im Hotel einen Skandal vermeiden wolle, wobei er ihm gleichzeitig Ratschläge gibt, wie er es am besten unangenehmen Kontrollen der Polizei entgehen könne.

Von Venedig reist der Ich-Erzähler schließlich mit einem alten Passagierdampfer auf die der dalmatinischen Küste vorgelagerte Insel Ceratosa, einen Ort, den der Erzähler nur deshalb als Bestimmungsort ausgewählt hat, weil ihm das Fahrgeld fehlte, um bis nach Ragusa zu gelangen. Diese Insel hat nichts gemeinsam mit der Mondänität der Côte d'Azur oder des Lido von Venedig. Das einzige Hotel im Ort sieht aus wie « eine Festung aus verputztem Stein " (21) ist altmodisch und sehr provinziell. Dennoch gelingt es ihm als Lord Chesterton sehr schnell, Bekanntschaft mit den wenigen im Hotel anwesenden vornehmen Vertretern der höheren Gesellschaft zu machen, unter anderem mit dem Fürsten Balearu aus Bukarest und dessen Frau sowie mit einem ehemaligen Offizier namens von Rakic, den aber der Erzähler sofort als Hochstapler ausmacht. Die meisten Hotelbewohner hält er allerdings für « Kleinwild », Leute, die er, anders als etwa in Venedig, nicht um besonders viel Geld erleichtern können wird: "Kleinwild, dem nicht mehr Geld in der Tasche saß, als für die laufende und die nächste Hotelrechnung nötig war. Kleinwild aus Agram, Belgrad und Budapest. Balkan. Ich schaute mir das an - geärgert und belustigt zugleich. » (22)

Ein wesentliches Merkmal des Hochstaplers ist das Verschleiern seiner wahren Identität. Der Ich-Erzähler tritt fast an jedem Ort unter einem anderen Namen auf, wobei Bemerkungen wie "ich nannte mich auf dem Formular Mr. Davis aus London " (9) erkennen lassen, daß es sich nie um seinen richtigen Namen handelt. Sogar am Ende der Novelle, als er sich gezwungen sieht, um einem Duell zu entgehen, brieflich zu gestehen, daß er nicht der wirkliche Lord Chesterton sei, unterschreibt er diesen Brief nicht mit seinem Namen, sondern nur mit « der Ihre ». Seine wahre Identität und seine 
wirkliche soziale Abstammung bleiben genauso im Dunkeln wie die Frage nach seiner Muttersprache.

15 Sein privilegiertes «Einsatzgebiet» ist der Mittelmeerraum, jene mondänen Fremdenverkehrsorte, die seit dem Aufkommen des Tourismus Treffpunkt des europäischen Geburts und Geldadels geworden sind : Er ist an der Côte d'Azur genauso zu Hause wie an der italienischen Riviera oder am Lido von Venedig und an der dalmatinischen Küste, wobei Frankreich und Italien den Vorzug des Ich-Erzählers vor den dalmatinischen Inseln haben, die er verächtlich als «Balkan » abtut und auf denen er sich eben nur deshalb aufhält, weil es für ihn zu diesem Zeitpunkt keine andere Möglichkeit gibt, seiner Beschäftigung nachzugehen.

Der Erzähler betrachtet die Hochstapelei als eine Tätigkeit wie jede andere, als Arbeit, um seinen Lebensunterhalt zu verdienen : Es geht darum, Geschäft zu machen. Immer wieder muß er sich das in Erinnerung rufen, daß er, «Lord Chesterton, [...] nicht zu [s]einem Vergnügen hierher gekommen » (36) sei.

17 Seinem angeblichen Stand entsprechend und gemäß dem von ihm vorgegebenen Reichtum geht der Erzähler meistens zwei Beschäftigungen nach : Er versucht, Frauen zu verführen, denen er wertvolle Geschenke machen will, welche er sich auf betrügerische Weise erschwindeln muß; doch meistens vertreibt er sich die Zeit mit dem Glücksspiel: Es ist seine Hauptbeschäftigung und sein hauptsächlicher Gelderwerb, wobei seine Präferenz dem Kartenspiel gilt, das er durch Falschspielen jederzeit beeinflussen kann. Ein Ort gewinnt erst seine Attraktivität, wenn er über ein Spielcasino verfügt. Nie geht er ohne eine «Karte in der Manschette» (12) an den Spieltisch, von dem er magisch angezogen ist. So wird ein Ausflug mit dem Segelboot von Ceratosa zum nahegelegenen Ruinenort Lianora, der vor nicht allzu langer Zeit von seinen Bewohnern verlassen worden ist, weil dort die Pest gewütet hat, erst richtig interessant, als die Organisatoren den Teilnehmern eröffnen, daß es in dem Ort auch die Möglichkeit zu spielen gebe. Ganz nebenbei will der Ich-Erzähler dort noch die Fürstin Balearu verführen.

18 Nach der Überfahrt findet sich ein halbes Dutzend Männer in einem Raum des halb verfallenen Schlosses von Lianora zum Kartenspiel ein. Dieser ist ein Spiegelbild sowohl des Glückspiels als auch der Hochstapelei, wo der Schein mehr gilt als die Wirklichkeit. In dem Raum befinden sich nämlich «Bilder [...], die waren Türen; Türen, die waren Spiegel ; Spiegel, die waren nur aufgemalt oder blind » (48), doch nichts ist da, was es zu sein scheint. Gleichzeitig herrscht durch das Kerzenlicht, das die Köpfe der Anwesenden in Totenschädel verwandelt, eine Stimmung des Untergangs, die durch das regelmäßige Geschrei eines Totenvogels noch verstärkt wird.

Beim Kartenspiel, das fast die ganze Nacht bis zum Morgengrauen dauert, geht es dem Ich-Erzähler in erster Linie nicht darum zu gewinnen, sondern er will seine Mitspieler und den Verlauf des Spiels kontrollieren. Er muß seinen Konkurrenten von Rakic in die Schranken verweisen, auch wenn er anfangs Skrupel hat, gegen einen "Kollegen » vorzugehen; vor allem aber will er durch sein (Falsch) Spiel die Fäden ziehen, bestimmen, wer gewinnt und wer verliert und dabei das Gefühl seiner Macht über die anderen genießen - das Ziel, sich selbst zu bereichern, tritt dabei in den Hintergrund. So gehen durch die Spielzüge des Ich-Erzählers der ortsansässige Pope und der deutsche Professor Wendelin, ein Antikeforscher, mit sehr viel Geld vom Spieltisch, während der neureiche Geschäftsmann Marcelli und der Hochstapler von Rakic fast völlig ruiniert sind. Als der Ich-Erzähler kurze Zeit später von Rakic wieder begegnet, 
steht er einem menschlichen Wrack gegenüber, das kaum mehr menschliche Züge aufweist :

Auf dem Wagen lag ein Paket, in eine Decke gehüllt. Das Paket hatte einen Kopf. Herr von Rakic! Seine Augen waren auf mich geheftet. Er wollte die Lippen bewegen, aber sie zitterten nur ganz leicht. Nein nichts regte sich an dem Verpackten. Nur seine Augen waren furchtbar unbeirrt und schimmernd auf mich geheftet - ein Abgrund des Lebens. (92) kommt es endlich zu dem vom Ich-Erzähler ersehnten Rendez-vous zwischen der Fürstin Balearu und ihm. Sie sind aber kaum zusammen, als der Fürst in die Suite des falschen Lord Chesterton eindringt, dort seine Frau vorfindet und deswegen Genugtuung fordert : Im Morgengrauen des kommenden Tages wollen sich die beiden Männer zum Duell treffen. In dieser Situation ist der Ich-Erzähler, und das ist ein weiterer Charakterzug des Hochstaplers, aber nicht gewillt, jenen Pflichten nachzukommen, die sich aus dem Ehrenkodex der Gesellschaftsschicht ergeben, der anzugehören er vorgibt. Um das Duell nicht bestreiten zu müssen, ist er sogar bereit, in einem Brief seine wahre Identität preiszugeben. Doch es kommt nicht zum Äußersten : Es stellt sich nämlich heraus, daß Balearu ebenfalls nicht die Absicht hat, sich mit dem Ich-Erzähler zu schießen, da er genauso wenig ein rumänischer Fürst ist wie dieser ein englischer Lord. Der Hochstapler ist selbst Opfer eines anderen, aus Sachsen stammenden Hochstaplers namens Liebschlögel geworden. Beide müssen in einem Gespräch unter vier Augen ihre falsche Identität aufgeben und sich gegenseitig eröffnen, in Wirklichkeit Betrüger zu sein.

Am Ende der Novelle zieht der Ich-Erzähler eine eher triste Bilanz: « Da saß man also tatsächlich auf dem Trockenen. Aber gut angezogen war man immerhin, und man war gewitterkühl bis ins Herz. Und klaren Hirns. » (93) Er hat jetzt nicht mehr Geld als er bei seiner Ankunft besessen hat, ja er verfügt nicht einmal über genügend Geldmittel, um die Insel verlassen zu können. So ist er gezwungen, seine Rolle weiterzuspielen. Ohne Perspektive auf eine Änderung seiner Verhältnisse kehrt er erneut an den Spieltisch des Hotels zurück, in der Absicht, mit den Neuankömmlingen Geschäfte zu machen und sie um ihr Geld zu bringen, diesmal aber gemeinsam mit Liebschlögel und seiner Partnerin :

Was will man - Geschäft ist Geschäft ! [...] Der dicke Mann mit den Ringen ging auf den Leim. Ein Viertelstündchen - da stand ich an seinem Tisch. Ein Viertelstündchen - da hatte ich ihn mit Fürst und Fürstin bekannt gemacht. Ein Viertelstündchen - da saßen wir drunten im Spielsalon, und ich fächerte die Karten über die Platte. [...] Oh, er hatte der Karten nicht sonderlich acht. As und sieben hatte ich in der Manschette. Und Cœur-Dame in der Fuge unter dem Tisch. (94)

Robert Neumann löst in seiner Hochstaplernovelle alle zur Figur des Hochstaplers gehörenden Klischees ein, ja er überzeichnet sie so stark, daß er sie ad absurdum führt. Wie absurd in Wirklichkeit das Spiel mit dem Schein und dem Sein ist, eröffnet die Episode, in der der eine Hochstapler den anderen betrügen will. Neumann sieht den Hochstapler als einen Glücksritter, der es nie zu Reichtum bringen wird, jedoch seine 
Misere hinter dem Schein des vorgegebenen hohen Standes versteckt. An seinem Lebenswandel hält er aus Prinzip fest und ist gleichzeitig Gefangener der sich daraus ergebenden Prinzipien, denen er nur im äußersten Notfall untreu wird. Die eindimensionale, subjektive Darstellung, die zwar als Studie eines bestimmten Typus besticht, gerät aber schließlich wegen der fehlenden Distanz des Subjekts zu seinem Tun zur Karikatur.

In diesem Zusammenhang drängt sich die Frage auf, inwieweit die Zeichnung des Hochstaplers auf den Schriftsteller Robert Neumann zurückwirkt. Die subjektive Darstellung von "Karrieren », von Menschentypen, die Neumann im Grunde fremd sein müssen, kann nichts anderes sein als die Darstellung von auf allgemeine Merkmale reduzierte Typen, also auf eine Wiedergabe des Scheins, so daß Neumann unter diesem Gesichtspunkt völlig zuzustimmen ist, daß es sich bei ihm als Schriftsteller des Roman und Novellenzyklus Karrieren um einen Hochstapler handelt.

\section{NOTES}

1. In Thomas Manns Roman Bekenntnisse des Hochstaplers Felix Krull ist Olympia Felix Krulls Schwester und findet bereits auf der ersten Seite Erwähnung.

2. Eine ausführliche Darstellung der Auseinandersetzung zwischen Robert Neumann und Erika Mann ist nachzulesen in: Irmela von der Lühe : Erika Mann. Eine Biographie. Frankfurt/Main. Fischer Taschenbuch 1996, S. 345-349.

3. Erika Mann : Mit eigener Feder. In : Das Schönste VII. Jg. Nr.7, 1961, S. 32 f. Zitiert nach : von der Lühe : Erika Mann, S. 347.

4. Thomas Mann: Bekenntnisse des Hochstaplers Felix Krull. Buch der Kindheit. Wien, Leipzig, München, Rikola 1922.

5. Robert Neumann : Karrieren. München, Verlag Kurt Desch 1966, S. 399.

6. Thomas Mann : Briefe. Hg. Erika Mann, Frankfurt/Main, Fischer 1962-1965.

7. Robert Neumann : Vielleicht das Heitere. Tagebuch aus einem anderen Jahr. München, Wien, Basel, Verlag Kurt Desch 1968, S. 592.f.

8. Rolf Düsterberg : «Mein Reichsführer, lieber Heini Himmler ». In : Die Zeit, Nr. 12, 11.3.2004.

9. Klaus Mann war Chefredakteur und Herausgeber der Exilzeitschrift Die Sammlung, einer Monatsschrift, die im Amsterdamer Querido Verlag in den Jahren 1933-1935 erschien und zu deren Autoren unter anderem Bert Brecht, Alfred Döblin, Heinrich Mann, André Gide, Aldous Huxley, Josef Roth und Arnold Zweig gehörten.

10. Cf. Rolf Düsenberg.

11. Es handelt sich dabei um die Romane und Novellen Hochstaplernovelle, Karriere, Blinde Passagiere, Luise, Olympia und Mathilde Walewska.

12. Friedrich Nietzsche: Menschliches, Allzumenschliches, In : F.N. : KSA 2, hg. v. Giorgio Colli und Mazzino Montinari, München, Berlin, New York, dtv/de Gruyter 1988, S. 71 f.

13. Robert Neumann : Hochstaplernovelle. In : Robert Neumann : Karrieren. München, Verlag Kurt Desch 1966. In der Folge wird bei Zitaten nur mehr die Seitenzahl des Textes angegeben. 


\section{RÉSUMÉS}

In seinem Prosatext Hochstaplernovelle (1930), dem ersten von sechs Romanen und Novellen, in denen Robert Neumann verschiedene Menschentypen von einer subjektiven Erzählhaltung her beschreiben will, gestaltet er den Typ eines Hochstaplers, wobei er sich vor allem darauf beschränkt, dessen typische Merkmale - der ständige Wechsel der Identität, das andauernde Spielen einer Rolle, die Vorgaukelung eines Scheins - herauszuarbeiten, ohne daß der Hochstapler sich je in Frage stellt, so daß seine Darstellung zur Karikatur wird.

Le texte Hochstaplernovelle (1930) est la première nouvelle d'une série de six romans et nouvelles, dans laquelle Robert Neumann tente de décrire différents types d'homme dans une perspective subjective, il dépeint le portrait de l'imposteur.

Cependant, Neumann se limite à la description de ses traits typiques tels que le changement permanent de l'identité, le jeu d'un rôle etc. sans que l'imposteur ne mette jamais en cause son existence d'imposteur. De ce fait, la représentation devient caricature.

\section{AUTEUR}

\section{ALFRED STRASSER}

Université Charles-de-Gaulle - Lille 3 